\title{
A DISFAGIA NO ENVELHECIMENTO ASSOCIADA A DESNUTRIÇÃO E DESENCADEAMENTO DE TRANSTORNOS MENTAIS
}

\section{The dysphagia in the aging associated with mental disorder and malnutrition}

Suelen Capelari ${ }^{1}$ Josiane Budni ${ }^{2 *}$

1Fonoaudióloga, mestranda pelo programa de pós graduação em Ciências da Saúde Universidade do Extremo Sul Catarinense - UNESC, Criciúma, SC, Brasil.

'Laboratório de Doenças Neurodegenerativas; Laboratório de Neurociências; Programa de Pós-Graduação em Ciências da Saúde; Unidade Acadêmica de Ciências da Saúde -Universidade do Extremo Sul Catarinense - UNESC, Criciúma, SC, Brasil.

* Autor correspondente: Laboratório de Neurociências, Programa de Pós-

Graduação em Ciências da Saúde, Unidade Acadêmica de Ciências da Saúde, Universidade do Extremo Sul Catarinense, 88806-000 Criciúma, SC, Brazil;

E-mail: josiane.budni@unesc.net

Tel.: +55 (48) 3431-2792 


\section{Resumo}

Considerando o declínio da homeostase do organismo causado pelo envelhecimento, esta revisão de literatura tem como objetivo contribuir para o aprofundamento de informações e aperfeiçoamento dos profissionais da saúde sobre o processo envelhecimento e suas comorbidades de forma a proporcionar maior longevidade com qualidade à população que está envelhecendo. $O$ diagnósticos de demências cresce em todo mundo e associado à elas, a disfagia, o indica uma maior probabilidade para a desnutrição, o que é relevante para os aspectos físicos, funcionais, sociais e econômicas. Assim, a desnutrição está associada com maior risco de mortalidade, taxas mais elevadas de infecção, um aumento do número de quedas e fraturas, permanência hospitalar, má qualidade de vida, ansiedade e depressão. Ao realizar tal levantamento de dados pode-se observar que há escassos estudos que correlacionam envelhecimento, depressão, déficit cognitivo com disfagia dos idosos. O aumento acelerado da população idosa, torna necessário mais pesquisas sobre o envelhecimento.

Palavras-chave: Envelhecimento; Depressão; Demência; Disfagia

\section{Abstract}

Considering the decline of the homeostasis of organism caused by aging, this literature review aims to contribute to the deepening of information and improvement of health professionals about the aging process and its comorbidities in order to provide greater longevity with quality to the population that is growing old. The diagnosis of dementias are increasing worldwide and associated with them, the dysphagia, indicates a greater probability for malnutrition, which is relevant for the physical, functional, social and economic aspects. Therefore, the malnutrition is associated with a higher risk of mortality, higher infection rates, an increase in the number of falls and fractures, overstaying in hospital, poor quality of life, anxiety and depression. On the research, we could perceive that there are few studies that correlate aging, depression, cognitive deficit with dysphagia of the elderly. The 
Artigo Original

Fisiopatologia

accelerated increase of the elderly population, makes researching about aging more substantial than ever.

Keywords: Aging; Depression; Dementia; Dysphagia.

\section{INTRODUÇÃO}

Há um aumento da população na faixa etária de 60 anos ou mais na maioria dos países em desenvolvimento, segundo a Organização Mundial da Saúde (OMS), nos países em desenvolvimento, se considera idoso o indivíduo com idade igual ou superior a 60 anos, e nos países desenvolvidos, o indivíduo com idade igual ou superior a 65 anos (1). A Pesquisa Nacional de Amostra por Domicílio (2) de 2011 demonstra que o Brasil possui cerca de 23,5 milhões de habitantes acima dos 60 anos. As estimativas indicam que em 2025, existirão cerca de 1,2 bilhões de pessoas com idade igual ou superior a 60 anos. Isso representará um quinto da população mundial, e fará com que o Brasil seja a 6ª nação com o maior número de idosos do mundo, com perspectivas superiores a 30 milhões de pessoas $(3,4,1,5)$. 0 que explica essa crescente são medidas tomadas pelo governo federal que visam a melhoria da qualidade de vida do idoso, e também a ampliação da expectativa de vida dos brasileiros, que pulará de 75 anos em 2013 para 81 anos em 2060 (5).

A longevidade carrega consigo grandes preocupações, uma vez que esta pode ser acompanhada por uma série de dificuldades que podem interferir negativamente na qualidade de vida dessa população (3).

O envelhecimento representa uma etapa normal do desenvolvimento do indivíduo onde há adaptações em as funções do organismo, por modificações celulares, teciduais e morfofisiológicas.

Assim, considerando o cenário nacional que se dirige para uma quantidade cada vez maior de pessoas idosas na população, e que esses idosos podem, em sua maioria, apresentar múltiplas doenças crônicas e que estas podem causar certo grau de dependência, este século será caracterizado por novas necessidades de cuidado $(6,7)$. 


\section{O ENVELHECIMENTO}

O envelhecimento pode ser conceituado como um processo natural, dinâmico e progressivo que representa um aumento de fragilidade ocasionado pelas modificações fisiológicas, bioquímicas, morfológicas e psicológicas que acabam por gerar perda gradativa da capacidade de adaptação ao ambiente e aumento de dependência $(8,7,9)$.

A dependência pode ser um fator interferente, já que a mesma pode ser considerada uma condição vivida por pessoas que por perda ou falta de autonomia física, psíquica e/ou social, necessitam de auxilio na realização de atividades básicas e instrumentais da vida diária. Tal condição se torna um problema de saúde e interfere tanto na qualidade de vida do idoso como na de seu cuidador $(7,3)$.

Cerca de $50 \%$ dos idosos apresentam algum grau de dependência, como a compra e o preparo dos alimentos, uso de medicamentos, transportes, limpeza da casa e o controle das finanças (10). A dependência funcional está intimamente relacionada com aspectos de saúde, como aumento de doenças crônicas, declínios cognitivos e níveis de estresse (3). O estudo da prevalência de doenças associadas ao processo de envelhecimento é de grande importância sob o ponto de vista cognitivo e funcional, com o objetivo de prevenir e/ou minimizar os efeitos degenerativos próprios do envelhecimento, proporcionando maior qualidade de vida para o idoso (11).

O envelhecimento representa uma etapa do desenvolvimento do indivíduo onde há perdas na função normal do organismo e, portanto, déficits físicos, cognitivos e comportamentais que são provenientes de alterações biológicas em células e tecidos, aumentando, desta forma, o risco de desenvolvimento de doenças associadas ao envelhecimento, como por exemplo, a demência (12, 9). Com o envelhecimento, no cérebro ocorrem mudanças de várias esferas neurobiológicas e neurofisiológicas, como sinapses diminuídas, lentidão do fluxo axoplasmático, decréscimo na plasticidade, assim como mudanças neuroquímicas e estruturais (12). O declínio da capacidade cognitiva provém de processos fisiológicos normais do envelhecimento ou na transição para as demências, quadro em que a disfunção cognitiva é muito acentuada (9). 
Conforme a população envelhece, há um aumento na prevalência de déficits cognitivos, o que compõe um importante fator de risco para a saúde pública mental. Estas alterações cognitivas podem sofrer influências de fatores como prática de atividade física, idade, escolaridade e renda (13).

\section{A DEGLUTIÇÃO DO IDOSO}

Fazem parte do campo de atuação do profissional da área de fonoaudiologia as funções estomatognáticas (suç̧ão, mastigação, respiração, deglutição e fonoarticulação) e as estruturas a elas envolvidas (mandíbula, área oclusal, lábios, língua, bochechas, palato), dentre tais funções destacamos o processo da deglutição, que para seu bom funcionamento se faz necessário integridade e coordenação de um complexo grupo de estruturas interdependentes $(14,15)$.

A deglutição pode ser dividida em quatro fases de acordo com a região em que 0 fenômeno se desenvolve: oral, faríngea e esofágica $(16,17)$. A fase oral é dita voluntária e pode ser subdividida nos estágios de preparo, qualificação, organização e ejeção. O preparo se caracteriza por ser voluntário e se estender desde a escolha do alimento e do instrumento para a oferta até o tempo em que o alimento é triturado e misturado à saliva pela mastigação. A qualificação se dá em associação com o estágio de preparo, é caracterizada pela percepção do bolo, volume, consistência, densidade, grau de umidificação entre outras características. No estágio de organização, o bolo é posicionado sobre o dorso da língua e projetado para trás, através de movimentos ondulatórios da língua. Já a fase faríngea, é involuntária, inicia pelo disparo da deglutição e é seguida da elevação e anteriorização da laringe e da abertura do esfíncter esofágico superior para a passagem do bolo. Durante a fase faríngea, o escape nasal é impedido pelo fechamento do esfíncter velofaríngeo que é a adaptação do palato mole contra a parede posterior da faringe, evitando, a dissipação da pressão e a regurgitação nasal do alimento. De forma simultânea, inicia-se a sequência da contração da musculatura constritora da faringe em sentido crânio-caudal. Por fim, a fase esofágica (involuntária), é a fase em que o bolo é levado por movimentos peristálticos até o estômago. $(16,17)$. 
Estas fases envolvem diversas estruturas orofaciais, mole e duras. As estruturas duras são os ossos: hióide, esfenóide, maxilar, mandíbular e vértebras cervicais. Os tecidos moles são compostos por músculos e outros tecidos, a orofaringe, músculos constritores faríngeos, palato mole, bochechas, língua (musculo genioglosso, hioglosso e estiloglosso), epiglote, esôfago, cartilagens cricóide e tireóide, e os músculos do pescoço (18).

Dentre os nervos que inervam tais estruturas envolvem, o trigêmeo ( $\mathrm{V}$ par craniado, nervo motor e sensitivo), Facial (VII par craniano, nervo motor e sensitivo), Glossofaríngeo (IX par craniano, nervo motor e sensitivo), Vago (X par craniano, nervo motor e sensitivo), Hioglosso (XII par craniano, nervo motor) e espinhal Cervical (nervo motor) (18).

A faringe se estende desde a base do crânio até a o nível da sexta vértebra cervical, medindo cerca de $12 \mathrm{~cm}$, e é dividida em três áreas anatômicas: a nasofaringe, orofaringe e a hipofaringe. É constituída de três músculos, estes, envolvidos no ato da deglutição: constritores da faringe superior, médio e inferior (18).

A laringe é constituída de cartilagens, músculos e ligamentos. As cartilagens, tireóide, cricóide, aritenóide, cuneiforme, corniculada e epiglote. Os músculos intrínsecos da laringe delimitam os movimentos de abertura e fechamento das pregas vocais durante a respiração, a fonação e deglutição (18).

A laringe tem como função primária a respiração, mas desenvolveu a fonação como função secundária, além de participar no mecanismo de proteção das vias aéreas inferiores durante o processo de deglutição. Sabe-se que as pregas vocais verdadeiras além da fonação, são as estruturas mais eficazes de proteção contra a aspiração $(18,17)$.

O esôfago é um tubo muscular envolto com uma mucosa que propulsiona o alimento da hipofaringe ao estômago. O músculo cricofaríngeo ou esfíncter esofageal superior formam a junção entre a hipofaringe e o esôfago. O esfíncter gastroesofágico ou esfíncter esofágico inferior forma a junção entre o esôfago e o estômago. Estes dois esfíncteres conservam o esôfago vazio entre as deglutições $(19,18)$. A faringe e o esôfago proximal são as únicas regiões do corpo humano 
onde a musculatura estriada não está sob o controle neurológico voluntário. Ambas as fibras, simpática e parassimpáticas, inervam o esôfago $(19,18)$.

Alterações em qualquer fase da deglutição, que trazem dificuldade para a passagem do bolo alimentar da cavidade oral até o estômago, induzem graves consequências que podem levar à desnutrição e até à morte, por comprometer a integridade das vias aéreas $(16,14,20)$. A disfagia, além de danos metabólicos, pode influenciar no estado emocional do paciente, causar estresse, depressão e isolamento social $(14,21)$.

Adaptações neste processo são características próprias do envelhecimento, onde ocorre uma série de modificações morfofisiológicas, destas estruturas, e de processos em que envolvem as mesmas (22). À medida que se envelhece, a capacidade de deglutir de forma segura passa por modificações fisiológicas e anatômicas que podem beneficiar o risco de uma deglutição descoordenada, o que pode gerar complicações como má nutrição e desidratação, além de ser um dos principais fatores causadores de pneumonias em idosos (21, 23). Consequentemente, estas alterações podem contribuir para o aumento dos índices de morbidade. Uma das justificativas para essas alterações é a ocorrência da diminuição do controle do sistema neuromuscular, podendo resultar no prejuízo de qualquer uma das fases da deglutição $(21,23)$.

Designa-se presbifagia a adaptação no ato de deglutir do idoso saudável, caracterizada pela condução do bolo alimentar, da boca até o estômago, que acontece no envelhecimento $(24,25)$. A presbifagia faz parte do envelhecimento natural e fisiológico do mecanismo de deglutição. $(22,24)$.

\section{PRESBIFAGIA E TRANSTORNOS MENTAIS}

Mais de 35 milhões de pessoas apresentam algum grau de demência, este número poderá triplicar até 2050 atingindo mais de 115 milhões de pessoas (26).

O diagnósticos da doença de Alzheimer ou outras demências cresce dramaticamente em muitos países, associado à elas, a disfagia se faz presente nos estágios mais avançados da doença o indica uma maior probabilidade estar em risco 
de desnutrição. Entende-se por desnutrição a desordem ou diminuição na ingestão ou absorção de nutrientes com relação às necessidades individuais $(27,28)$.

A maioria dos idosos com disfagia (cerca de $70 \%$ ) recebem dieta com textura modificada, por exemplo, em pacientes com disfagia para líquidos, recomenda-se produtos artificiais como espessantes alimentares sem sabor, ou via alimentar alternativa como a sonda nasoenteral (SNE) (29, 27, 28). Doenças neurodegenerativas produzem alterações no nível de consciência ou no mecanismos de deglutição o que muitas vezes obriga um suporte nutricional especializada, tanto no hospitalar e em casa (29).

Sabe-se que a disfagia, diagnóstico comum entre pessoas idosas, pode resultar em desnutrição e aumentar o risco de pneumonia o que é uma causa comum de mortalidade em pacientes com demência e outros distúrbios cognitivos $(30,31)$. Disfagia piora consideravelmente estado nutriconal do idoso o que é particularmente relevante para as consequências físicas, funcionais, sociais e econômicas, assim, a desnutrição está associada com maior risco de mortalidade, taxas mais elevadas de infecção, um aumento do número de quedas e fraturas, permanecia hospitalar, má qualidade de vida, ansiedade e depressão $(32,29,28$, 33).

Nos estudos de Yang (30) pode-se observar que a presença do transtorno depressivo maior é três vezes maior em pacientes disfágicos, onde $45 \%$ dos individos achavam que comer era agradável, $41 \%$ sentiam ansiedade ou pânico durante as refeições, e $36 \%$ evitam comer por disfagia, assim, os autores trazem que há impacto psicológico provocado pela disfagia em pacientes idosos.

Além disso, em idosos, alguns fatores como prejuízos no ambiente sócio econômico também deixam o indivíduo susceptível ao desenvolvimento de transtornos psiquiátricos, dentre estes transtornos, a depressão também é a que mais se destaca, comprometendo a qualidade de vida do idoso de forma direta (34).

A depressão maior é um transtorno mental que ocupa a quarta posição da lista mundial de incapacidade e é esperado que se torne o segundo transtorno mais frequente até 2030 (35; 36; 37). Os atuais critérios de diagnóstico do DSM-V-TR incluem humor deprimido e/ou anedonia por pelo menos quinze dias, além de sintomas adicionais como preocupação excessiva, sentimento de culpa, pensamento 
suicida, alterações psicomotoras e de sono, aumento ou diminuição do peso corporal, modificação do apetite e alterações cognitivas (38).

Considerando, que a depressão apresenta alterações biológicas semelhantes ao processo do envelhecimento e a de doenças neurodegenerativas, ser frequentemente associada à doença cardiovascular, às demências no geral e à demência do tipo doença de Alzheimer (DA), faz com que os mecanismos fisiopatológicos associados aos transtornos de humor continuem sendo um desafio para a sociedade científica. Isso contribui para a dificuldade de tratamento adequado destes indivíduos (39, 40, 41).

O cérebro é conhecidamente suscetível ao estresse oxidativo, e este parece desempenhar importante papel na doença de Alzheimer, identificou-se em estudos $(42,43)$ que pacientes com DA apresentavam consumo inadequado de antioxidantes, como vitaminas $A, C, E, B 12$, zinco e selênio por serem disfágicos.

Níveis mais baixos de ácido fólico fazem relação com menor desempenho cognitivo, memória e velocidade psicomotora. Sabe-se que suplementação de vitamina B12 e de acido fólico pode reduzir a progressão da doença e melhorar o desempenho cognitivo dos pacientes com DA $(42,43)$.

Contudo a se faz necessário um olhar de prevenção e intervenção precoce para assegurar um suporte nutricional adequado em consistência ou via alimentar segura para o idoso de modo a retardar o avanço da doença e promover maior qualidade de vida, para tanto é importante a abordagem multidisciplinar o que permitirá incluir as contribuições de diferentes domínios profissionais (44).

\section{CONCLUSÃO}

Levando em consideração o aumento acelerado da população idosa e os poucos estudos sobre a relação entre envelhecimento, depressão, déficit cognitivo e disfagia dos idosos, tornam-se necessárias mais pesquisas sobre o envelhecimento e disfagias. Este assunto é de extrema importância, no intuito de contribuir à crescente demanda por procedimentos diagnósticos, terapêuticos e de reabilitação nesta população e por ser fundamental que os profissionais e cuidadores de saúde estejam capacitados para diagnosticar e monitorar a evolução dos sintomas. Isso 
poderia diminuir o indice de desnutrição, uso de vias alternativas de alimentação, pneumonia broncoaspirativas e interações de repetição. Assim, poderia contribuir para uma melhor qualidade de vida dos idosos.

\section{REFERENCIAS}

1. Carvalho VL de. Perfil das instituições de longa permanência para idosos situadas em uma capital do Nordeste. Cad. saúde colet. 2014;22(2):184-191.

2. BRASIL. Secretaria de Atenção à Saúde. Departamento de Atenção Básica. (2006c). Envelhecimento e saúde da pessoa idosa (Cadernos de Atenção Básica, 19). Brasília, DF.

3. Melo RLP de et al. Sentido de vida, dependência funcional e qualidade de vida em idosos. Rev. bras. geriatr. gerontol. 2013;16(2):239-250.

4. Silva JMN da et al. Correlação entre o risco de queda e autonomia funcional em idosos institucionalizados. Rev. bras. geriatr. gerontol. 2013;16(2):337-346.

5. Melo NCV de et al. Household arrangements of elderly persons in Brazil: analyses based on the national household survey sample (2009). Rev. bras. geriatr. gerontol. 2016;19(1):139-151.

6. Pavarini $\mathrm{SCl}$ et al. A arte de cuidar do idoso: gerontologia como profissão?. Texto contexto - enferm. 2005;14(3):398-402.

7. Marinho LM et al. Grau de dependência de idosos residentes em instituições de longa permanência. Rev. Gaúcha Enferm. 2013;34(1):104-110.

8. Albino ILR et al. Influência do treinamento de força muscular e de flexibilidade articular sobre o equilíbrio corporal em idosas. Rev. bras. geriatr. gerontol. 2012;15(1):17-25.

9. Trindade APNT da et al. Repercussão do declínio cognitivo na capacidade funcional em idosos institucionalizados e não institucionalizados. Fisioter. mov. 2013;26(2):281-289.

10. Oliveira LPBA de, Menezes RMP de. Representações de fragilidade para idosos no contexto da estratégia saúde da família. Texto contexto - enferm. 2011;20(2):301-309.

11. Santos CCC dos et al . Análise da função cognitiva e capacidade funcional em idosos hipertensos. Rev. bras. geriatr. gerontol. 2011;14(2):241-250. 
12.SANTOS, Flávia Heloísa dos; ANDRADE, Vivian Maria and BUENO, Orlando Francisco Amodeo. Envelhecimento: um processo multifatorial. Psicol. estud. 2009, vol.14, n.1, pp. 3-10. ISSN 1413-7372.

13. Domiciano BR et al. Cognitive function of elderly residents in long-term institutions: effects of a physiotherapy program. Rev. bras. geriatr. gerontol. 2016;19(1):57-70.

14. Marcolino J. Achados fonoaudiológicos na deglutição de idosos do município de Irati - Paraná. Rev. bras. geriatr. gerontol. 2009;12(2):193-200.

15. Etges $\mathrm{CL}$ et al . Screening tools for dysphagia: a systematic review. CoDAS, 2014;26(5):343-349.

16. Yamada, EK et al. A influência das fases oral e faríngea na dinâmica da deglutição. Arq. Gastroenterol. 2004;41(1):18-23.

17. Cintra $A B$ et al. Deglutição após quimioterapia e radioterapia simultânea para carcinomas de laringe e hipofaringe. Rev. Assoc. Med. Bras. 2005;51(2):93-99.

18. Marchesan IQ. Deglutição - Normalidade. "In": Furkim AM, Santini CS. Disfagias Orofaríngeas. São Paulo: Profono; 1999.3-18.

19. Pinheiro NL et al. Estudo ultra-estrutural do esôfago de Trichogenes longipinnis. Britski \& Ortega - Pisces, Siluriformes, Trichomicteridae. Rev. Bras. Zool., 1993;10(4):629-632.

20. Pires EC, Sassi FC, Mangilli LD, Limongi SCO, Andrade CRF. Alimentos na consistência líquida e deglutição: uma revisão crítica da literatura. Rev. soc. bras. Fonoaudiol.; 2012;17(4):482-488.

21. Furkim AM, Duarte ST, Hildebrandt PT, Rodrigues KA. A instituição asilar como fator potencializador da disfagia. Rev. CEFAC. 2010; 12(6):954-963.

22. Lima RMF et al. Adaptações na mastigação, deglutição e fonoarticulação em idosos de instituição de longa permanência. Rev. CEFAC [online]. 2009;11(3):405-422.

23. Magalhaes Jr, Hipólito $V$ et al . Caracterização da pressão da língua em idosos. Audiol. Commun. Res. 2014;19(4):375-379.

24. Cassol $\mathrm{K}$ et al. Qualidade de vida em deglutição em idosos saudáveis. J. Soc. Bras. Fonoaudiol. 2012; 24(3):223-232.

25. Oliveira BS de, Delgado SE, Brescovici SM. Alterações das funções de mastigação e deglutição no processo de alimentação de idosos institucionalizados. Rev. bras. geriatr. gerontol. 2014;17(3):575-587. 
26. Dias $G$ et al. Atividades avançadas de vida diária e incidência de declínio cognitivo em idosos: Estudo SABE. Cad. Saúde Pública, Rio de Janeiro. 2015; 31(8):1623-1635.

27. Vaca Bermejo R, Ancizu García I, Moya Galera D, de las Heras Rodríguez, Pascual Torramadé J. Prevalencia de desnutrición en personas mayores institucionalizadas en España: un análisis multicéntrico nacional. Nutr Hosp. 2015;

28. Abe K, Yamashita, Kondo K, Takayama K, Yokota O, Sato Y. Terada S. LongTerm Survival of Patients Receiving Artificial Nutrition in Japanese Psychiatric Hospitals. Dementia and Geriatric Cognitive Disorders EXTRA. 2016;6(3), 477485.

29. Luis DA, Izaola O, Fuente B, Muñoz-Calero P, FrancoLopez A. Enfermedades neurodegenerativas; aspectos nutricionales Nutrición Hospitalaria. Grupo Aula Médica Madrid, España. 2015;32(2): 946-951.

30. Yang EJ, Kim MH, Lim J, Paik N-J. Oropharyngeal Dysphagia in a CommunityBased Elderly Cohort: the Korean Longitudinal Study on Health and Aging. Journal of Korean Medical Science. 2013;28(10):1534-1539.

31.Nimmons, D., Michou, E., Jones, M., Pendleton, N., Horan, M., Hamdy, S. A Longitudinal Study of Symptoms of Oropharyngeal Dysphagia in an Elderly Community-Dwelling Population. Dysphagia. 2016; 31 560-566.

32. Alvarez-Hernandez J, Leon-Sanz M, Planas Vila M, Araujo K, Garcia de Lorenzo A, Celaya-Pérez S, On behalf of the PREDyCESR researchers. Prevalence and costs of malnutrition in hospitalized dysphagic patients: a subanalysis of the PREDyCES® study. Nutr Hosp. 2015;32(4):1830-1836.

33. Ayres A, Jotz G P, Rieder C R M, Schuh A F Sr, Olchik, M R. The Impact of Dysphagia Therapy on Quality of Life in Patients with Parkinson's Disease as Measured by the Swallowing Quality of Life Questionnaire (SWALQOL). International Archives of Otorhinolaryngology. 2016; 20(3): 202206.

34. Almeida L, Quintão S. Depression and suicidal ideation in elderly institutionalized and non-institutionalized in Portugal. Acta Med Port. 2012;25(6):350-8.

35. Forlenza OV, Almeida OP. Depressão e Demência no Idoso - Tratamento Psicológico e Farmacológico. São Paulo: Lemos.1997.

36. Gold PWR, Vieira M, Pavlatou MG. Clinical and Biochemical Manifestations of Depression: Relation to the Neurobiology of Stress. Neural Plasticity. 2015;2015 Article ID 581976:11 pages. 
37. Vaváková M, Ďuračková Z, Trebatická J. Markers of Oxidative Stress and Neuroprogression in Depression Disorder. Oxidative Medicine and Cellular Longevity. 2015;2015Article ID 898393:12 pages.

38. American Psychiatric Association -APA. DSM-V-TR: manual diagnóstico e estatístico de transtornos mentais -versão revisada.5 Ed.Porto Alegre:Artmed, 2014.

39. Leonardo BE. Psychopathology of Depression. Drugs Today (Barc). 2007;43(10):705-16.

40. Wolkowitz OM, Reus VI, Mellon SH. Of sound mind and body: depression, disease, and accelerated aging. Dialogues Clin Neurosci. 2011;13:25-39.

41. Maes M, Mihaylova I, Kubera M, Uytterhoeven M, Vrydags N, Bosmans E. Increased 8-Hydroxy- Deoxyguanosine, a Marker of Oxidative Damage To DNA, in Major Depression and Myalgic Encephalomyelitis / Chronic Fatigue Sýndrome. Neuro Endocrinol Lett. 2009;30(6):715-22.

42. Goes VF, Mello-Carpes PB, Oliveira LO de, Hack J, Magro M, Bonini, JS. Avaliação do risco de disfagia, estado nutricional e ingestão calórica em idosos com Alzheimer. Revista Latino-Americana de Enfermagem. 2014;22(2):317-324.

43. ALMEIDA CC, BRENTANI HP, FORLENZA OV, DINIZ B S. Serum folic acid is reduced in patients with Alzheimer's disease. Rev. psiquiatr. clín 2012;39(3):9093.

44. BAENA GONZÁLEZ M, MOLINA RECIO G. Abordaje de la disfagia en enfermos de alzhéimer. Nutrición Hospitalaria.2016;33(3):739-748. 